\title{
Canaliculitis masquerading as Dacryocystitis: Rhinologist's Dilemma
}

\author{
${ }^{1}$ Nishi Gupta, ${ }^{2}$ Sima Das, ${ }^{3}$ Neeraj Chawla
}

\section{ABSTRACT}

Aim: To highlight canaliculitis masquerading as chronic dacryocystitis that may mislead a rhinologist.

Background: Canaliculitis is an inflammation of the lacrimal canaliculi caused by infection or as a complication of punctal plug insertion.It is often misdiagnosed, leading to a delay in diagnosis.

Case report: We report a case of a 20 -year-old female patient who presented with complaints of watering from left eye since 1 year and was referred to the Department of Ear, Nose, and Throat for endoscopic dacryocystorhinostomy (DCR). She had a history of silicone punctal plug insertion in the same eye for "dry eye" following an attack of herpes zoster 12 years earlier. She was asymptomatic for 10 years following punctal plug insertion, but started complaining of epiphora since the last 2 years. Punctal plug was removed 1 year back by an ophthalmologist but the epiphora persisted. Regurgitation of purulent discharge on pressure over lacrimal sac area made it look like dacryocystitis. Syringing through lower punctum was blocked while through the upper punctum it was patent. Thus, possibility of nasolacrimal duct block was ruled out. An occuloplasty opinion was sought and a diagnosis of lower canaliculitis with possibility of retained plug was made.

Conclusion: Canaliculitis should be considered as a differential diagnosis in cases presenting with epiphora as canaliculitis is a close masquerade of dacryocystitis.

Clinical significance: As more and more rhinologists are getting direct referrals of cases needing endoscopic DCRs, it is important for them to be well aware of other associated pathologies of proximal lacrimal system. This will help them to reach a correct diagnosis before going ahead with endoscopic DCR.

Keywords: Canaliculitis, Dacryocystitis, Epiphora, Masquerading.

How to cite this article: Gupta N, Das S, Chawla N. Canaliculitis masquerading as Dacryocystitis: Rhinologist's Dilemma. Clin Rhinol An Int J 2017;10(2):91-92.

\section{Source of support: Nil}

\section{Conflict of interest: None}

\footnotetext{
${ }^{1}$ Associate Medical Director and Head, ${ }^{2}$ Consultant and Head ${ }^{3}$ Consultant

1,3 Department of ENT, Dr. Shroff's Charity Eye Hospital, New Delhi, India

${ }^{2}$ Department of Oculoplasty, Dr. Shroff's Charity Eye Hospital New Delhi, India

Corresponding Author: Nishi Gupta, Associate Medical Director and Head, Department of ENT, Dr. Shroff's Charity Eye Hospital, New Delhi, India, Phone: +919811898107, e-mail: nishibgupta@gmail.com
}

\section{BACKGROUND}

Canaliculitis is an inflammation of the lacrimal canaliculi caused by infection or as a complication of punctal plug insertion. ${ }^{1}$ It is often misdiagnosed leading to a delay in diagnosis. ${ }^{2}$ Canaliculitis is an uncommon condition, often can mimic many other common ocular conditions. It should be appropriately diagnosed and treated to avoid recurrent inflammation and possible obstruction of the upper portion of the lacrimal system. ${ }^{1}$

It is of utmost importance for rhinologists to be well versed with conditions of the proximal lacrimal pathway, such as canaliculitis since it can masquerade as chronic dacryocystitis. Careful clinical examination of the medial can thus alert the physician to that diagnosis.

\section{CASE REPORT}

We report a case of a 20-year-old girl who presented with complaints of watering from left eye since 1 year and was referred to the Department of Ear, Nose, and Throat (ENT) for endoscopic dacryocystorhinostomy (DCR). She had history of silicone punctal plug insertion for dry eye following an attack of herpes zoster 12 years ago. She was asymptomatic for 10 years and started having epiphora since the last 2 years. Punctal plug was removed 1 year back by an ophthalmologist but the epiphora persisted. Regurgitation of discharge on pressure over lacrimal sac area made it look like dacryocystitis. Ocular examination did not show any apparent swelling, redness, or induration in the lacrimal area.

Syringing through lower punctum was blocked, while through the upper punctum it was patent. Thus, possibility of nasolacrimal duct block was ruled out and a diagnosis of lower canaliculitis was made.

Three-snip punctoplasty with canalicular curettage was done (Fig. 1). Yellow granular material (Fig. 2) was removed from the lower canaliculus. Material was sent for culture and microbiological examination. Microbiological examination revealed Actinomyces sp. Though, on culture, no growth was seen as culturing of Actinomyces is difficult due to its anaerobic nature. ${ }^{1}$ It was concluded that concretions which do not grow characteristic findings of Actinomyces or other organism may simply be sterile necrotic tissue and debris. 


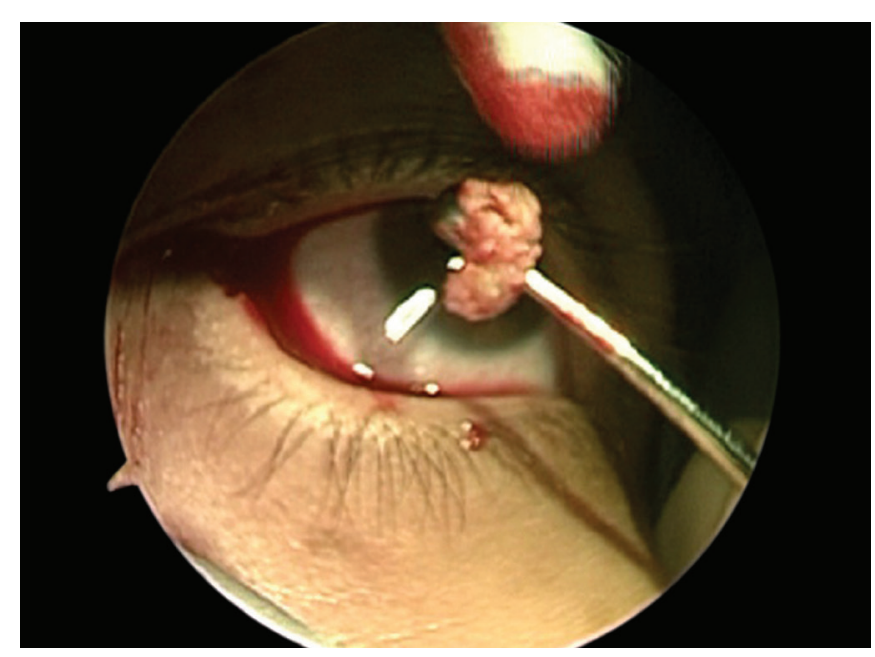

Fig. 1: Concretions from canaliculi

Syringing was repeated through lower canaliculi postsurgery and was found to be patent. On 6 months follow-up, patient was free of symptoms.

\section{DISCUSSION}

Canaliculitis can masquerade as chronic dacryocystitis in patients presenting with epiphora and may pose a clinical challenge for a rhinologist. Advantage of working in an eye and ENT hospital gives us an opportunity to see a variety of such cases and thus enables in better understanding of different conditions causing epiphora.

Lacrimal canaliculitis often remains undiagnosed for extended periods of time as a result of its rarity. Patients with canaliculitis typically present with epiphora, punctal discharge, and a pouting punctum; however, they often masquerade as a chronic purulent conjunctivitis, hordeolum, chalazion, or dacryocystitis leading to unnecessary manipulations and further delay in the effective treatment of this condition. ${ }^{2}$

If the literature accurately reflects clinical practice, it would appear that it is more common to misdiagnose patients with canaliculitis than to identify this condition. Case studies of canaliculitis report percentages of patients previously misdiagnosed as $45 \%$ in a major review by Freedman et $\mathrm{al}^{3}$ in 2011. In a study by Baldursdóttir et al, ${ }^{4}$ at least seven of the nine subjects with canaliculitis had been misdiagnosed previously.

Chronic lacrimal canaliculitis is rarely detected in clinical practice, accounting for $2 \%$ of lacrimal duct diseases. Of the agents involved in canaliculitis, Actinomyces varieties, Gram-positive, anaerobic bacteria that are difficult to isolate and identify are the most commonly isolated, although other bacteria, fungi, and viruses may also appear. ${ }^{5}$ The yellowish granular material removed by curettage in our case is the yellow dacroliths called "sulfur granules" and is typical of Actinomyces sp.

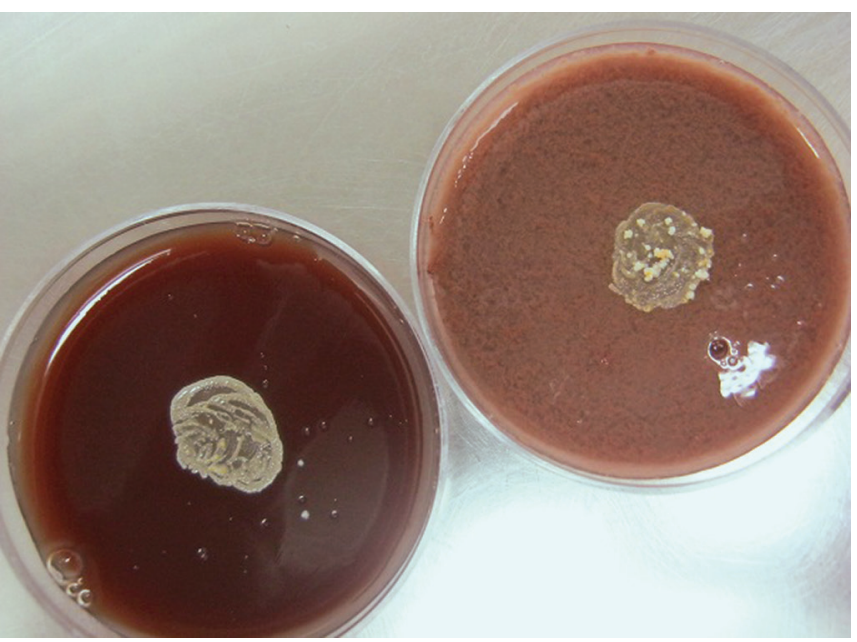

Fig. 2: Yellowish granules (sulfur granules) in a petri dish

Decision regarding management depends on the final diagnosis. In our case, three-snip punctoplasty along with curettage of canaliculi led to complete cure, but resistant or recurrent cases associated with nasolacrimal duct block may need DCR as well.

\section{CONCLUSION}

Canaliculitis is a rare condition but a close masquerade of dacryocystitis. It should be considered as a differential diagnosis in all cases presenting with epiphora and especially in patients with history of previous lacrimal procedures like silicone punctal plug insertion.

\section{CLINICAL SIGNIFICANCE}

As more and more rhinologists are getting direct referrals of cases needing endoscopic DCRs, it is important for them to be well aware of other associated pathologies of proximal lacrimal system. This will help them to reach a correct diagnosis before going ahead with endoscopic DCR.

\section{REFERENCES}

1. Zaveri J, Cohen AJ. Lacrimal canaliculitis. Saudi J Ophthalmol 2014 Jan;28(1):3-5.

2. Lin SC, Kao SC, Tsai CC, Cheng CY, Kau HC, Hsu WM, Lee SM. Clinical characteristics and factors associated the outcome of lacrimal canaliculitis. Acta Ophthalmol 2011 Dec;89(8):759-763.

3. Freedman JR, Markert MS, Cohen AJ. Primary and secondary canaliculitis: a review of literature. Surv Ophthalmol 2011 Jul-Aug;56(4):336-347.

4. Baldursdóttir E, Sigurdsson H, Jónasson L, Gottfredsson M. Actinomycotic canaliculitis: resolution following surgery and short topical antibiotic treatment. Acta Ophthalmol 2010 May;88(3):367-370.

5. Yılmaz MB, en E, Evren E, Elgin U, Yılmazba P. Canaliculitis awareness. Turk J Ophthalmol 2016 Jan;46(1):25-29. 